\title{
ZnMgO-based UV photodiodes: a comparison of films grown by spray pyrolysis and MBE
}

\author{
A. Hierro*a , G. Tabares ${ }^{\mathrm{a}}$, M. Lopez-Ponce ${ }^{\mathrm{a}}$, J.M. Ulloa ${ }^{\mathrm{a}}$, A. Kurtz ${ }^{\mathrm{a}}$, E. Muñoz ${ }^{\mathrm{a}}$, V. Marín-Borrás ${ }^{\mathrm{b}}$, \\ V. Muñoz-Sanjoséb, J.M. Chauveau ${ }^{c}$
}

a ISOM-Dept. Ingenieria Electronica, Univ. Politecnica de Madrid, Avda. Complutense 30, 28040 Madrid, Spain; ${ }^{b}$ Dpto. de Física Aplicada y Electromagnetismo, Universidad de Valencia, Spain;

'CRHEA-CNRS, 06560 Valbonne, France, and University of Nice Sophia Antipolis, Parc Valrose, 06102 Nice cedex 2, France

\begin{abstract}
Detecting the UV part of the spectrum is fundamental for a wide range of applications where ZnMgO has the potential to play a central role. The shortest achievable wavelength is a function of the Mg content in the films, which in turn is dependent on the growth technique. Moreover, increasing $\mathrm{Mg}$ contents lead to an electrical compensation of the films, which directly affects the responsivity of the photodetectors. In addition, the metal-semiconductor interface and the presence of grain boundaries have a direct impact on the responsivity through different gain mechanisms. In this work, we review the development of ZnMgO UV Schottky photodiodes using molecular beam epitaxy and spray pyrolysis, and we analyze and compare the physical mechanisms underlying the photodetector behavior.
\end{abstract}

Keywords: ZnO, ZnMgO, photodetector, UV, MBE, spray pyrolysis

\section{INTRODUCTION}

ZnO has a bandgap that lies in the near UV and thus can play a leading role in light photodetection in this spectral region. Indeed, UV photodetection includes a wide range of applications, which may be grouped in two areas: UV astrophysics and astronomy, and UV terrestrial applications. The first group includes several applications such as solar and astronomical UV imaging, and secure space-to-space communications (both inter- and intra-satellite). The second group includes monitoring of sun UV light exposure of the population, biological and chemical sensors (ozone, pollution in air, biological agents, etc.) and fire/flame detection (fire alarm systems, combustion engine control, missile plume detection).

To cover both the near UV (down to $300 \mathrm{~nm}$ ) and mid UV (down to $200 \mathrm{~nm}$, including the solar blind region), the ternary $\mathrm{ZnMgO}$ needs to be used. By adding $\mathrm{Mg}$ to $\mathrm{ZnO}$, the bandgap can be potentially tuned from 3.37 up to $6.2 \mathrm{eV}$ with a wurtzite structure. However, as it was already shown in the early 2000's with different growth techniques (MBE [1], PLD [2] and MOCVD [3]), increasing the Mg content eventually produces a phase separation, and the cubic structure appears, where $\mathrm{MgO}$ is stable. It is this maximum $\mathrm{Mg}$ content before phase separation that determines the minimum achievable cutoff wavelength. Moreover, the appearance of phase separation, as well as the presence of crystal defects, directly limit the performance of the ZnMgO UV photodiodes (PD). We compare here PDs realized on material grown by two very different techniques: molecular beam epitaxy (MBE) and spray pyrolysis (SP), where the maximum achievable $\mathrm{Mg}$ is quite different, and where the physical mechanisms limiting the responsivity are also quite different.

*adrian.hierro@upm.es;

Oxide-based Materials and Devices VII, edited by Ferechteh H. Teherani, David C. Look,

David J. Rogers, Proc. of SPIE Vol. 9749, 97490W · @ 2016 SPIE

CCC code: $0277-786 X / 16 / \$ 18 \cdot$ doi: $10.1117 / 12.2213697$ 


\section{EXPERIMENTAL DETAILS}

The ZnMgO structures grown by MBE were deposited on r-plane sapphire (Fig. 1). The MBE-grown ZnMgO structures were $1 \mu \mathrm{m}$-thick, with a a-plane orientation. Details of the growth can be found in [4]. The stoichiometry, substitutional character of Mg, and crystallinity was assessed by a combination of Rutherford Backscattering Spectroscopy (RBS), channeling RBS, and XRD [5]. The Mg content shown in Table 1 was extracted from these measurements, and the Mg substitutional character was confirmed for almost all Mg contents, whereas no cubic intrusions where observed. Thus, the single wurtzite crystal character was confirmed up to 56\% Mg. Moreover, the RBS channeling analysis shows a yield that is comparable to that from state of the art $\mathrm{ZnO}$ substrates, indicative of the high crystal quality of the films.

The SP films were deposited on a-sapphire with a 200-500 nm thick, and a c-plane orientation Details on the growth can be found in [6]. The Mg content in these films could not be properly quantified by RBS due to the fact that they show some porosity. Thus, EDX spectroscopy was used instead, yielding the values shown in Table 1.

The metal layers were deposited by e-beam evaporation under high vacuum conditions. In the MBE-grown material, semitransparent Au-Schottky photodiodes $100 \AA$-thick using circular structures of diameter $200 \mu \mathrm{m}$ were fabricated coplanar to the ohmic contacts, which consisted of an annealed $1000 \AA$-thick Ti/Al/Ti/Au layer. The samples were exposed to a $\mathrm{H}_{2} \mathrm{O}_{2}$ pre-treatment to passivate the surface, which has been previously shown to lead to very high quality Schottky contacts on (Zn,Mg)O/ZnO PDs [7]. In the SP-grown material, metal-semiconductor-metal (MSM) contacts were realized with $800 \AA$-thick Au layers [8]. In this case, no $\mathrm{H}_{2} \mathrm{O}_{2}$ pre-treatment was used because of the high porosity of the films. For the responsivity analysis, a $1000 \mathrm{~W}$-Xe lamp with a $1 / 4 \mathrm{~m}$ monochromator were used to excite the photodiodes.

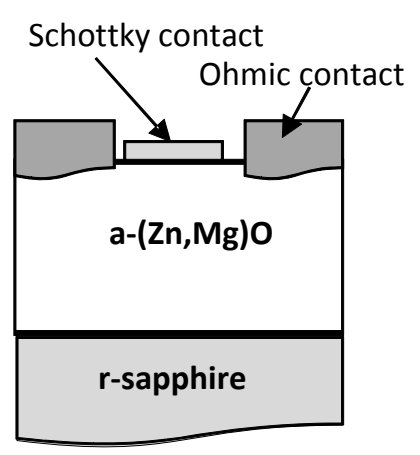

(a)

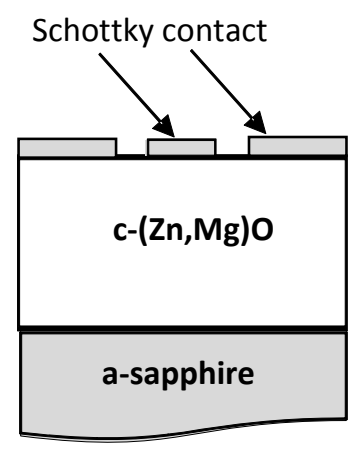

(b)

Figure 1. Schematic of the Schottky (a) and MSM (b) photodiodes on the MBE and SP layers, respectively. 
Table 1. Details of the photodiodes, their growth technique and the measured Mg content.

\begin{tabular}{|c|c|c|}
\hline Growth technique & Sample & $\mathbf{\% ~ M g}$ \\
\hline \multirow{4}{*}{ Molecular Beam Epitaxy } & MBE1 & 0 \\
\cline { 2 - 3 } & MBE2 & 31 \\
\cline { 2 - 3 } & MBE3 & 44 \\
\cline { 2 - 3 } & MBE4 & 52 \\
\cline { 2 - 3 } & MBE5 & 56 \\
\hline \multirow{4}{*}{ Spray Pyrolysis } & SP1 & 0 \\
\cline { 2 - 3 } & SP2 & 6 \\
\cline { 2 - 3 } & SP3 & 8 \\
\cline { 2 - 3 } & SP4 & 14 \\
\cline { 2 - 3 } & SP5 & 22 \\
\cline { 2 - 3 } & SP6 & 30 \\
\cline { 2 - 3 } & SP7 & 35 \\
\hline
\end{tabular}

\section{RESULTS AND DISCUSSION}

\subsection{MBE-grown Schottky UV photodiodes}

A detailed analysis of the IV curves from the photodiodes under dark conditions shows that the diodes have ideality factors between 1.2 and 1.8, Schottky barriers above $1.0 \mathrm{eV}$ and low reverse saturation currents in the $10^{-8}-10^{-11} \mathrm{~A} / \mathrm{cm}^{2}$ range. In addition, the rectification ratio between forward and reverse biases is as high as $10^{7}-10^{9}$ [9].

Table 2. Details of the PD figures of merit, including Schottky barrier $\left(\Phi_{b}\right)$, ideality factor $(n)$, series resistance $\left(R_{s}\right)$, reverse saturation current $(\mathrm{J}(-2 \mathrm{~V}))$, and current rectification ratio $(\mathrm{J}(+2 \mathrm{~V}) / \mathrm{J}(-2 \mathrm{~V}))$.

\begin{tabular}{|c|c|c|c|c|c|}
\hline Photodiode & $\boldsymbol{\Phi}_{\mathbf{b}}(\mathbf{e V})$ & $\mathbf{n}$ & $\mathbf{R}_{\mathbf{s}}(\mathbf{\Omega} \mathbf{c m})$ & $\begin{array}{c}\mathbf{J}(-2 \mathbf{V}) \\
\left(\mathbf{A} / \mathbf{c m}^{2}\right)\end{array}$ & $\mathbf{J}(+\mathbf{2 V}) / \mathbf{J}(-\mathbf{2 V})$ \\
\hline MBE1 & 1.2 & 1.2 & 1.78 & $-2 \times 10^{-9}$ & $\sim 10^{8}$ \\
\hline MBE2 & 1.2 & 1.4 & 5.52 & $-1 \times 10^{-8}$ & $\sim 10^{8}$ \\
\hline MBE3 & 1.2 & 1.6 & 16.00 & $-1 \times 10^{-11}$ & $\sim 10^{9}$ \\
\hline MBE4 & 1.0 & 1.7 & 16.36 & $-6 \times 10^{-11}$ & $\sim 10^{8}$ \\
\hline MBE5 & 1.0 & 1.8 & 11.71 & $-3 \times 10^{-10}$ & $\sim 10^{7}$ \\
\hline
\end{tabular}

The spectral response of the photodiodes was analyzed and the cutoff energy of the detector determined. As shown in Fig. 2 the cutoff energy can be tuned from $3.3 \mathrm{eV}$ for the $\mathrm{ZnO}$ photodiode, up to $4.7 \mathrm{eV}$ (264 nm) by increasing the $\mathrm{Mg}$ 
content up to $56 \%$. These short wavelengths already are in the solar blind region of the spectrum. The high quality of the Schottky photodiodes is evident from the very large UV/VIS spectral rejection ratios that are in the $10^{4}-10^{6}$ range. Indeed, detail analysis of the crystal structure of these films [5] shows that there are no cubic intrusions up to 56\% $\mathrm{Mg}$, the Mg sits in a substitutional site even in the high Mg content films, and the RBS channel yield is close to the state of the art commercial single crystal $\mathrm{ZnO}$ substrates. Thus, the high crystal quality allows reaching the solar blind region while keeping the wurtzite structure. This high crystal quality is also responsible for the quite reasonable responsivities, which in the $56 \% \mathrm{Mg}$ photodiode is around $0.1 \mathrm{~A} / \mathrm{W}$ (i.e., with internal quantum efficiency below $100 \%$ ), with no indication of any internal gain.
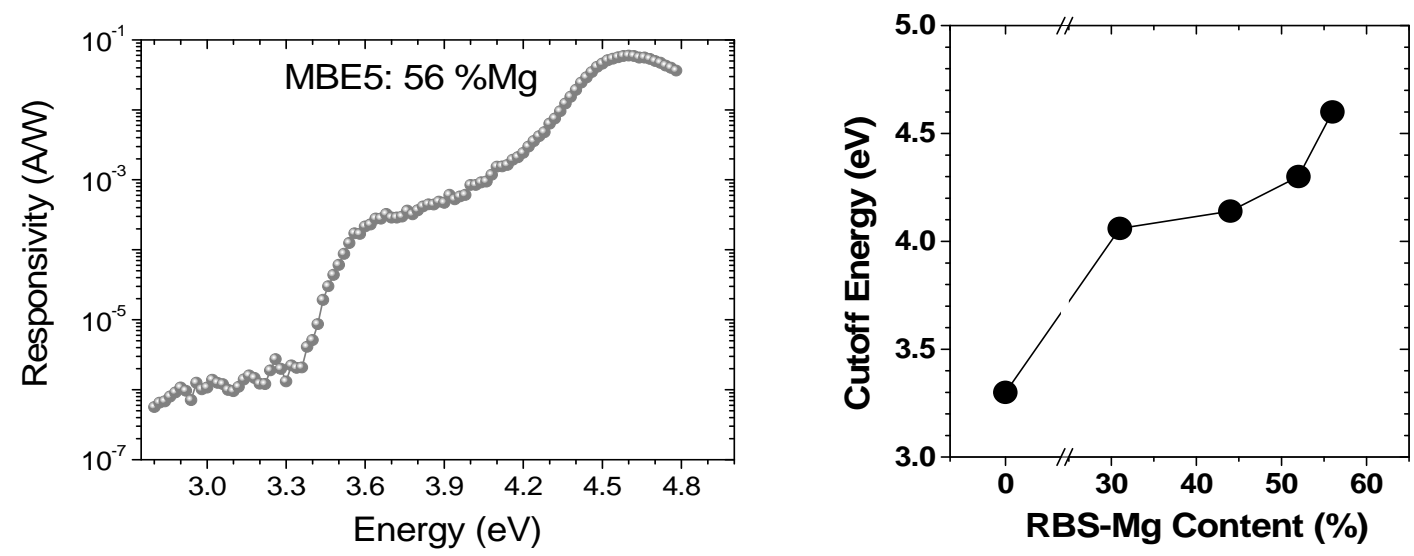

Figure 2. Left: Spectral response at $-1.5 \mathrm{~V}$ from the Schottky Photodiode with the highest Mg content. The feature at $3.4 \mathrm{eV}$ is the absorption from the $\mathrm{ZnO}$ buffer layer. Right: Cutoff energy of the Schottky photodiodes as a function of the Mg content in the film.

\subsection{SP-grown MSM UV photodiodes}

The SP-based MSM photodetectors show quite dark currents (Fig. 3), shifting from $10^{-3} \mathrm{~A}$ at $-4 \mathrm{~V}$ in the reference $\mathrm{ZnO}$ MSM, down to $10^{-9} \mathrm{~A}$ also at $-4 \mathrm{~V}$, in the $35 \% \mathrm{Mg}$ photodiode. This decrease in dark current with increasing Mg content is likely related to two factors. First, increasing the Mg content typically yields a lower carrier concentration in the ZnMgO film, producing more resistive layers [10]. Second, and most important in this case, the grain size decreases with increasing $\mathrm{Mg}$ content, yielding larger grain boundaries respect to the volume of the crystal [8]. This effect also directly affects the total carrier concentration and mobility of the films, such that films with small grains show much larger resistivities due to carrier trapping/scattering at the boundaries.

However, during illumination the photocurrent increases quite dramatically, yielding photocurrents in the $10^{-2}-10^{-3} \mathrm{~A}$ range, i.e., many orders of magnitude larger than the dark current for the high Mg MSM photodiodes. This illuminating/dark current ratios are thus in the $10^{1}$ range for $\mathrm{ZnO}$ and as high as $10^{7}$ for the high Mg MSM photodiode (see inset of Fig. 3). This is the result of above mentioned effect on the dark current, as well as the very high responsivities in these films, which are in the $10^{2}-10^{3} \mathrm{~A} / \mathrm{W}$ range. These responsivities yield internal quantum efficiencies well above $100 \%$, indicating a strong internal gain. This gain can be explained to arise from photo-generated carriers that are trapped at the grain boundaries, producing a strong photoconductive gain.

These MSM photodiodes made with SP films allows yielding cutoff energies up to $4.02 \mathrm{eV}$ (309 nm), but with very limited UV/VIS rejection ratios of only $10^{1}-10^{2}$. The large absorption tail observed below bandgap correlates quite well with the very large Stokes shift in these films, which increases with the Mg content up to $275 \mathrm{meV}$ for $35 \% \mathrm{Mg}$ [8]. However, as it was mentioned above, the extremely large illumination/dark current ratios above bandgap can be quite attractive for applications that require very high sensitivity but limited selectivity and response time, for a fraction of the price that is required to make an MBE-based UV photodiode. 

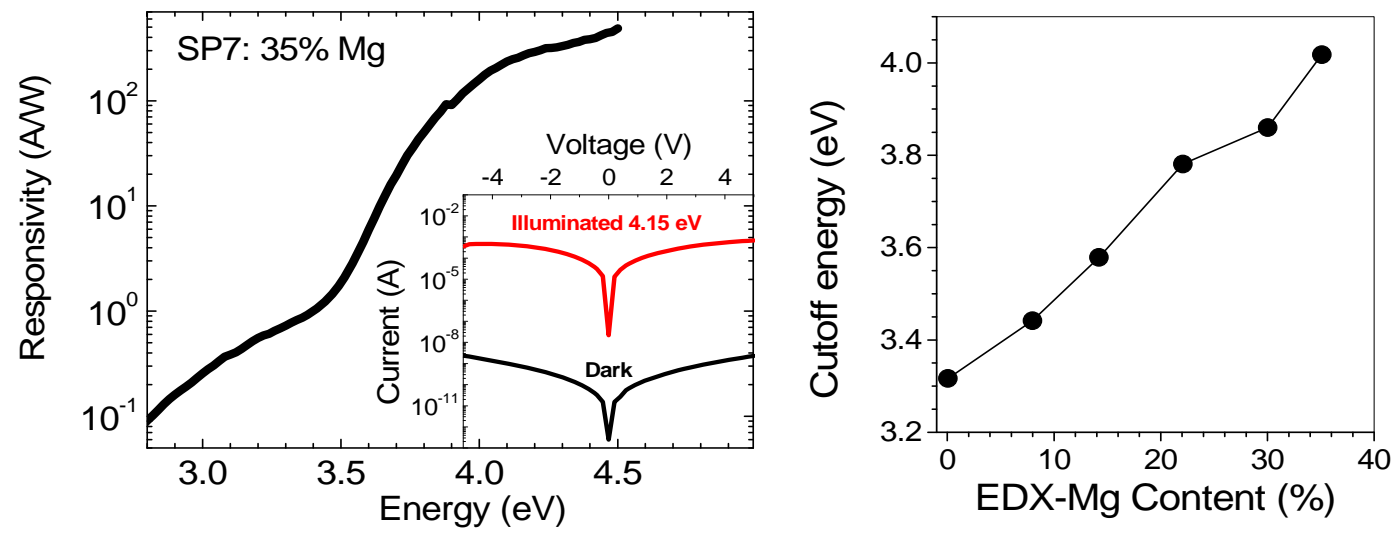

Figure 3. Left: Responsivity of SP-MSM photodiode at 4V with the highest Mg content. Right: Cutoff energy of the SPMSM photodiodes as a function of the Mg content in the film.

\section{CONCLUSIONS}

We have shown that both MBE and SP photodiodes can be used to cover part of the UV spectrum. With MBE ZnMgO films, the Mg concentration can be increased up to $56 \%$ without phase separation and high crystal quality, yielding absorption cutoff energies up to $4.7 \mathrm{eV}(264 \mathrm{~nm})$, in the solar blind region. These Schottky photodiodes have UV/VIS rejection ratios of up to $10^{6}$, with responsivities in the $0.1 \mathrm{~A} / \mathrm{W}$ for the highest $\mathrm{Mg}$ content, and no indication of internal gain. In contrast, SP allows incorporating Mg contents up to $35 \%$ before phase separation, yielding absorption cutoff energies around $4 \mathrm{eV}(309 \mathrm{~nm})$ for MSM photodiodes. The SP films are however polycrystalline, with grain sizes decreasing with the Mg content. This has a direct effect on the presence of a large internal photoconductive gain, which yields responsivities in the $10^{2}-10^{3} \mathrm{~A} / \mathrm{W}$. However, because of the very low dark current associated with the charge trapping/scattering at the grain boundaries, which happens in parallel to the large responsivities due to the photoconductive gain, produces illumination/dark current ratios as high as $10^{7}$, which is quite attractive for specific applications where a high sensitivity and low selectivity is needed.

\section{ACKNOWLEDGEMENTS}

This research was funded by the Spanish Ministry of Economy and Competitiveness (MINECO) through Projects No. TEC2011-28076-C02-01, TEC2014-60173-C2-2, and Grant No. BES-2012-051882; from the French National Agency through the young researcher project ANR 11-JS09-014 "HENOPOIN2"; and from the Generalitat Valenciana through projects Prometeo/2011-035 and ISIC/2012/008.

\section{REFERENCES}

[1] H. Tanaka, S. Fujita, and S. Fujita, "Fabrication of wide-band-gap MgxZn1-xO quasi-ternary alloys by molecular-beam epitaxy,” Appl. Phys. Lett. 86, 192911 (2005).

[2] W. Yang, R. D. Vispute, S. Choopun, R. P. Sharma, and T. Venkatesana, "Ultraviolet photoconductive detector based on epitaxial $\mathrm{Mg}_{0.34} \mathrm{Zn}_{0.66} \mathrm{O}$ thin films,” Appl. Phys. Lett. 78, 2787 (2001).

[3] W. I. Park, Gyu-Chul Yi and H. M. Jang, "Metalorganic vapor-phase epitaxial growth and photoluminescent properties of Zn1-xMgxO $(0 \leq x \leq 0.49)$ thin films,” Appl. Phys. Lett. 79, 2022 (2001). 
[4] J.-M. Chauveau, J. Vives, J. Zuniga-Perez, M. Laügt, M. Teisseire, C. Deparis, C. Morhain and B. Vinter, "Residual strain in nonpolar a-plane $\mathrm{Zn1}-\mathrm{xMg} \mathrm{xO},(0<\mathrm{x}<0.55)$ and its effect on the band structure of (Zn,Mg)O/ZnO quantum wells," Appl. Phys. Lett. 93, 232911 (2008).

[5] A. Redondo-Cubero, A. Hierro, J.-M. Chauveau, K. Lorenz, G. Tabares, N. Franco, E. Alves, and E. Muñoz, "Single phase «-plañe MgZnO epilayers for UV optoelectronics: substitutional behavior of $\mathrm{Mg}$ at large contents," Cryst. Eng. Comm. 14, 1637 (2012).

[6] S. Rajappan Achary, S. Agouram, J. F. Sanchez-Royo, M. Lopez-Ponce, J. M. Ulloa, E. Muñoz, A. Hierro and Vicente Muñoz-Sanjose, "Self-assembled $\mathrm{Mg}_{\mathrm{x}} \mathrm{Zn}_{1-\mathrm{x}} \mathrm{O}$ quantum dots $(0 \leq \mathrm{x} \leq 1)$ on different substrates using spray pyrolysis methodology,” Cryst. Eng. Comm. 15, 182 (2013).

[7] G. Tabares, A. Hierro, M. Lopez-Ponce, E. Muñoz, B. Vinter and J.-M. Chauveau, "Light polarization sensitive photodetectors with m- and r-plane homoepitaxial ZnO/ZnMgO quantum wells," Appl. Phys. Lett 106, 061114 (2015).

[8] M. Lopez-Ponce, A. Hierro, V Marín-Borrás, G. Tabares, A. Kurtz, S. Albert, S. Agouram, V MuñozSanjosé, E Muñoz and J M Ulloa, "Optical properties of ZnMgO films grown by spray pyrolysis and their application to UV photodetection,” Semicond. Sci. Technol. 30, 105026 (2015).

[9] E. Gür, G. Tabares, A. Arehart, J. M. Chauveau, and A. Hierro, "Deep levels in a-plane, high Mg-content MgxZn1-xO epitaxial layers grown by molecular beam epitaxy," J. Appl. Phys. 112, 123709 (2012).

[10] A. Hierro, G. Tabares, J. M. Ulloa, E. Mu noz, A. Nakamura, T. Hayashi, and J. Temmyo, "Carrier compensation by deep levels in $\mathrm{Zn}_{1-\mathrm{x}} \mathrm{Mg}_{\mathrm{x}} \mathrm{O}$ /sapphire,” Appl. Phys. Lett. 94, 232101 (2009). 